\title{
Transit Assignment Modeling Approaches based on Interval Uncertainty of Urban Public Transit Net Impedance
}

\author{
Qian TAN, Xiangdong ZHOU*, Wusheng LIU
}

\begin{abstract}
The data of the regular bus in Shenzhen during October 2019 was taken as an example. The improved model for the public transportation assignment was established based on considering the interval uncertainty theory and the basic algorithm of interval value, and the interval value acquisition method of bus impedance is established, the Method of Successive Averages (MSA) algorithm is used to solve the problem. Finally, the error analysis of bus passenger flow assignment before and after the improvement of the model is carried out. It is found that the average absolute percentage error of the improved assignment model is $8.7 \%$ compared with the real value, while the average absolute percentage error is $10.9 \%$ when the impedance is invariant value, The result of passenger flow assignment under interval impedance is obviously better than that under certain impedance. On non-working days, when the bus passenger flow changes greatly, the bus passenger flow assignment result under interval impedance is better.
\end{abstract}

Keywords: error analysis; improved model; interval uncertainty; transit assignment; travel time function

\section{INTRODUCTION}

The problem of urban traffic congestion is becoming more and more serious. Urban public transport is an effective way to alleviate traffic congestion. Reasonable urban public transport network design and scheduling is an important factor to improve the quality of public transport service and bus trip rate, and the allocation of public transport passenger flow is the basis of network design and scheduling optimization.

For transit assignment model, the current research is mostly based on the improvement of logit model. Dial [1, 2] put forward logit recursive model on the basis of original logit model, which regarded collinear lines as physical sections, and took the sum of departure frequency of all original collinear lines as operation frequency of combined lines. Chriqui and Robillard [3] analyzed bus passengers' choice behavior characteristics for the first time, and built the bus passenger flow assignment model according to this characteristic. Based on the collinear model proposed by Chiqui and Robillard [3], De Cea and Fernandez [4] put forward the mathematical expression of bus passenger flow assignment problem, that is, passengers' choice of travel path is composed of a series of transfer stations, and the new model can well deal with the problem of bus congestion. Lam et al. [5] considered the capacity limit of each transfer station, and used the logit based stochastic user equilibrium model. Based on the research of Cominetti and Correa [6], Cepeda et al. [7] proposed a bus passenger flow assignment algorithm on the grounds of departure frequency, which can be applied to large-scale network, and tested on real network. Poon et al. [8] assumed all passengers can get accurate travel information according to their travel experience, and by considering the generalized cost function of travel path selection, a schedule based equilibrium assignment model which obeyed first-in first-out principle under congested conditions is established. Hammouch [9] extended the model of Hammouch and Lawphongpanich [10] to distinguish the discomfort degree of sitting and standing passengers. Passengers of each category are grouped according to their remaining journey length and the time they have spent on the bus, and these probabilities are calculated by performing dynamic network loading. Zhang et al. [11] established a timetable based transit assignment model, in which they used the effective travel cost as a factor affecting the choice of passenger travel path, and considered the opportunity constraint of processing capacity. Codina [12] proposed a network equilibrium passenger flow distribution model at peak hours. Hamdouch considered the change of departure frequency for passenger flow assignment, and developed a dynamic transit assignment model based on schedule and departure frequency $[13,14]$. Sun and Szeto[15] proposed a logit bus passenger flow allocation model based on fixed demand.

In the research of interval number, Martorell's methods to deal with interval deterministic problems are mainly based on genetic algorithm, fuzzy sets and so on. Hugo Gilbert and Spanjaard [16] and Batarseh and Wang [17] respectively studied interval number and interval probability distribution. Salman Zaidi and Kroll [18] proposed a simplified interval calculation method. W. S. Liu combined with big data, studied the short-term prediction method of bus stop passenger flow and the value method of its uncertainty [19].

Generally speaking, the current research results can be classified into two categories, namely, equilibrium assignment model and multi-path probability assignment model. With the emergence of big data, few scholars use big data to assign public transport passenger flow. Many scholars believe that the use of big data can directly obtain the passenger flow travel path (OD) without assignment. In fact, the bus travel path can be obtained by using mobile signaling data, IC card data and GPS data. However, this path is based on the existing bus line network and station setting conditions, so, the passenger flow assignment is also generated on the existing bus line network. If the setting of the bus network is unreasonable, the passenger flow distribution is unreasonable, and it is difficult to optimize the line network according to the passenger flow distribution scheme. In addition, in the current assignment of public transport passenger flow, it is often considered that the number of passengers getting on and off at each station is a definite value. However, in fact, the number of bus passengers flow getting on and off at each station is often uncertain and fluctuates in a range. 
This paper combines the interval uncertainty theory with the bus passenger flow distribution, considers the interval uncertainty theory and the basic algorithm of the interval number. The logit model of the bus passenger flow assignment is constructed from the two aspects of the travel time function and the transfer time function of the bus passengers. The error analysis method of the bus passenger flow assignment before and after the model improvement is established, and morning peak, evening peak and offpeak periods are analyzed. The average error and maximum error of the bus line in the period of time.

\section{THE DEFINITION OF THE INTERVAL UNCERTAINTY OF BUS IMPEDANCE}

\subsection{Definition of Bus Impedance}

Transit impedance refers to the travel time, cost, convenience and other comprehensive indicators of passengers on the bus line. The conventional bus impedance model is as follows:

$R_{i j}=k_{1} T_{i j}+k_{2} W_{i j}$

where, $R_{i j}$ is the bus impedance of the path; $C_{i j}^{p}$ is the time impedance of the route, which generally refers to the total time required for passengers to complete a bus trip; $W_{i j}$ is the cost impedance of the route $i j$, which generally refers to the cost incurred by passengers to complete a bus trip. It is mainly related to the bus fare. $k_{1}, k_{2}$ is the coefficient of time impedance and cost impedance respectively.

In practice, because the economic cost of public transport travel is very low, the most important factor affecting the subjective evaluation of public transport travel mode is the travel time. In addition, the transfer penalty time is a kind of psychological resistance caused by the transfer of passengers, and the waiting time actually includes the transfer time. Therefore, this paper ignores the ticket price impedance and transfer penalty time, and the bus impedance only considers the in-car time and passenger boarding time.

(1) Travel time between bus stops

The bus stop travel time is the time that the bus stops from the stop $i$ to the stop $j$ of the stop.

In this paper, the time is the difference between the time when the vehicle leaves stop $i$ and the time when it arrives at stop $j$. The time when the bus leaves $i$ is the time when the bus arrives at $\mathrm{i}$ after the fusion of GPS data and IC card data. The time when the vehicle arrives at station $j$, is the time when the last passenger who gets on the bus at the station swipes the card.

$$
t_{i j}^{p}=t_{\mathrm{Aj}}-t_{\mathrm{B} i}
$$

where, $t_{\mathrm{A} j}$ is the time when the bus arrives at stop $j ; t_{\mathrm{B} i}$ is the time when the bus leaves stop $i$.

(2) Passenger boarding time

Joaquin defined the station boarding time in 1993 as [20]:

$t_{j}^{p}=\beta_{p}\left(\frac{v_{i p}}{K_{i p}}\right)^{\gamma p}$ where, $v_{j p}$ is the number of people on line $p$ at station $i ; K_{p}$ is the capacity of line $p$ (person/hour); $\beta_{p}, \gamma_{p}$ is the site congestion parameters.

Then the impedance function between bus stops is as follows:

$$
C_{i j}^{p}=t_{i j}^{p}+\beta_{p}\left(\frac{v_{i p}}{K_{i p}}\right)^{\gamma_{p}}
$$

\subsection{Analysis of Bus Impedance Uncertainty}

The bus impedance is affected by the traffic condition, the passenger flow of the station and other factors. For the same road section at different times, the bus running time has obvious differences, such as peak hours, due to traffic congestion, the bus running time between bus stops increases, and the bus impedance increases. There are also obvious differences in the passenger flow of stations in different periods of a day, and in working days and nonworking days, as well as in different months. Therefore, the running time of buses and the passenger flow at bus stops are obviously random, which leads to the value of urban bus impedance changing randomly over a period of time. In the past studies, the average value of the survey is often taken through the manual survey of sampling, which is difficult to express the actual bus impedance.

\subsection{Method of Obtaining Interval Uncertainty of Bus Impedance}

The research on the uncertainty of interval uncertainty of bus impedance started from the research of Bellman and Cooper in the mid-20th century [21]. In the optimization analysis, the traditional methods often regard the key parameters as a certain value. But in practice, the parameter is uncertain, which often brings some errors. At present, the main methods to deal with uncertain optimization problems include stochastic programming, fuzzy programming and non-probabilistic programming.

Interval number optimization method has some prominent advantages. First of all, stochastic programming and fuzzy programming need to determine the probability distribution or fuzzy membership function through limited data samples in practical application, so the solution results are deviated from the reality value. Interval number optimization method requires less data samples, and the concept of interval number is more simple and intuitive, which is easy to understand and apply.

In this paper, the interval value of bus impedance is obtained through big data analysis. First, multiple values of in-car time between adjacent stations are obtained. Then the quartile interval value of these data is calculated as the interval uncertainty value of the bus impedance. Quartile is a conception from statistics. To obtain the quartile, we need to arrange the data from small to large, and divide the sorted data into four equal parts. The value at the junction of each part is called the quartile. The first quartile $Q_{1}$ is called the lower quartile, the second quartile $Q_{2}$ is the median, and the third quartile $Q_{3}$ is the upper quartile.

Taking Shenzhen 325 line as an example, the line has 7 stations and 6 bus stops in Gongming central area. 2105 data are obtained in each section during the study time 
(October 11-10.31, 2019), of which 952 peak hours operation data are distributed as follows:
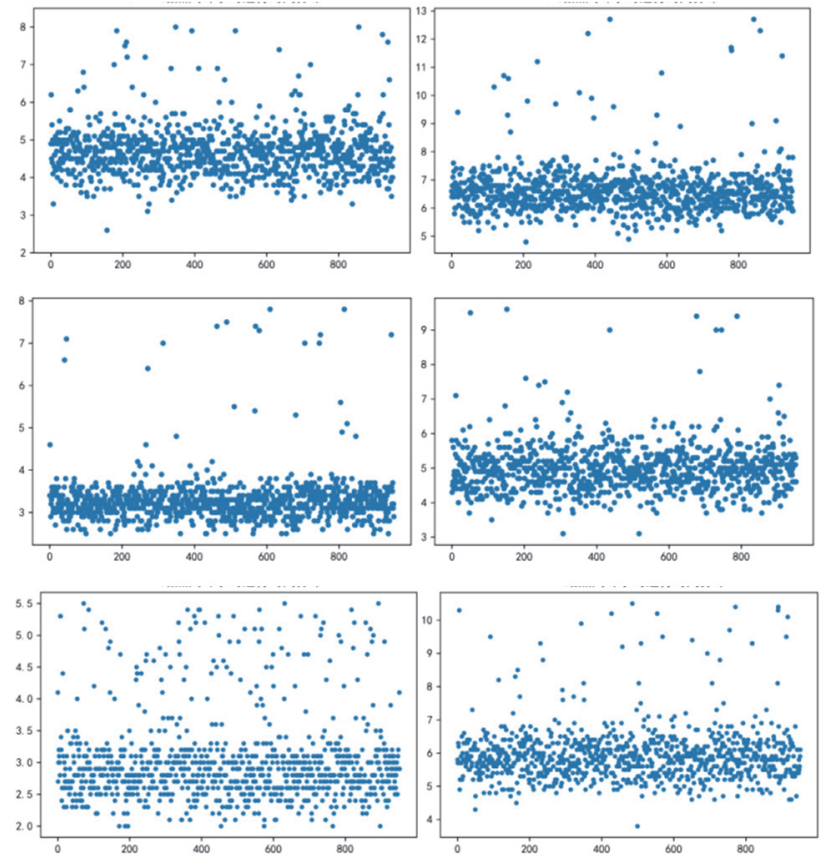

Figure 1 Peak hour operation time distribution of 325 bus stops

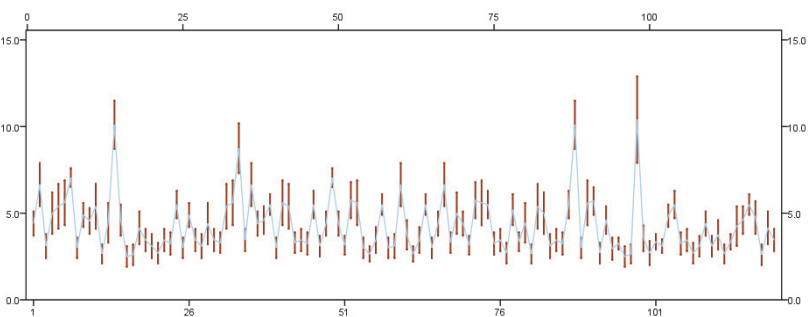

Figure 2 Time distribution of passenger in-car time between stations in peak hours in the study area

The ordinate in Fig. 1 is the peak hour operation time (minutes). The above six figures show the distribution of bus operation time at station 16 to station 15 , station 15 to station 3 , station 3 to station 1 , station 1 to station 2 , station 2 to station 4 and station 4 to station 17 respectively.

Take the quartile interval of the running time between each station to obtain the distribution of the time interval values of buses in peak hours in the study area, as shown in the figure. In Fig. 2, each line represents the running time between two stations, and the upper and lower ends of the line represent the upper and lower limits of the running time (minutes).

\section{MODEL UNDER INTERVAL IMPEDANCE \\ 3.1 Assumptions}

The model assumptions are as follows:

(1) The OD matrix of bus stops in peak time and offpeak periods time in the study area has been obtained.

(2) The bus passenger flow is only analyzed in this paper, therefore, only the bus passenger flow assignment is considered between the two traffic districts, and the passenger flow assignment of other traffic modes is not considered.
(3) The interference of special weather, holidays and major events are excluded. The hypothetical condition of this mode has considered the interference factors of traffic congestion, that is, the driving headway is equal to the departure headway.

\subsection{Model Formulations}

(1) Model under invariant impedance

SPIESS (1988) established the following bus passenger flow assignemt model when the impedance is invariant.

$$
\begin{aligned}
& \min \cdot z=\sum_{t \in D} \sum_{a \in A} c_{a t} v_{a t}+\sum_{t \in D} \sum_{i \in I} w_{i t} \\
& \sum_{a \in A_{i}^{+}} v_{a t}=\sum_{a \in A_{i}^{-}} v_{a t}+g_{i t}, \forall i \in N, \forall t \in D \\
& v_{a t} \leq f_{a} w_{i t}, \forall a \in A_{i}^{+}, i \in N, t \in D \\
& v_{a t} \leq 0, \forall a \in A, \forall t \in D
\end{aligned}
$$

where, $v_{a t}$ is the passenger flow with terminal point $t$ on the edge of $a$.

$c_{a t}$ is the passenger flow impedance with the terminal point $t$ on the edge $a$.

$w_{i t}$ is the waiting time of passenger flow with terminal $t$ at $i$ station.

$g_{i t}$ is the demand of passenger flow with terminal $t$ at $i$ station.

$f_{a}$ is the service frequency on the edge of $a$.

The model is a node based assignment method, and the assignment result is the passenger flow of different end points on each edge.

The duality of the model is as follows:

$$
\begin{aligned}
& \max \cdot z=\sum_{t \in D} \sum_{i \in I} g_{i t} \pi_{i t} \\
& \pi_{j t}+c_{a t}+\mu_{a} \geq \pi_{i t}, a=(i, j) \in A \\
& \sum_{a \in A_{i}^{+}} f_{a} \mu_{a}=1, i \in I
\end{aligned}
$$

$\mu_{a} \geq 0, a \in A$

where, $\pi$ is the dual variable corresponding to constraint (6); $\mu_{a}$ is the dual variable corresponding to constraint (7).

(2) Model under interval impedance

Referring to the robust deviation criterion of karasan et al. in 2001 [22], a bus passenger flow assignment model based on Minimax regret value is established.

Basic idea: two extreme scenarios are constructed, one is the worst scenario, the other is the best scenario under the scenario. The cost difference between the two scenarios is the maximum regret value, also known as robust cost. The problem of bus passenger flow assignement is transformed into the problem of robust cost minimization. 
The specific methods are as follows: firstly, all or no bus passenger flow is assigned, that is, each OD of passenger flow is assigned to the robust shortest path in the interval, and the upper bound of the used link impedance is taken as the calculation standard to obtain the worst scenario impedance of the path. Then, in this scenario, the lower bound value of the unselected road section is taken to get a road network with definite impedance, and the passenger flow is redistributed to get the best scenario impedance under this scenario. The cost difference between the worst and the best scenario is the maximum regret value or robust cost, and the optimization model is constructed with the objective of minimizing the robust cost.

Based on the robust deviation criterion and the minimum and maximum regret value as the objective, the bus passenger flow assignment model under the impedance of the interval road network is constructed as follows:

$$
\begin{aligned}
& \min \cdot z=\sum_{t \in D} \sum_{a \in A} \bar{c}_{a t} v_{a t}+\sum_{t \in D} \sum_{i \in I} w_{i t}-\sum_{t \in D} \sum_{i \in I} g_{i t} \pi_{i t} \\
& \sum_{a \in A_{i}^{+}} v_{a t}=\sum_{a \in A_{i}^{-}} v_{a t}+g_{i t}, \forall i \in I, \forall t \in D \\
& v_{a t} \leq f_{a} w_{i t}, \forall a \in A_{i}^{+}, i \in I, t \in D \\
& \pi_{j t}+\left[\overline{c_{a}} \delta_{a t}+\left(1-\delta_{a t}\right) \underline{c_{a}}\right]+\mu_{a} \geq \pi_{i t}, \\
& a=(i, j) \in A \\
& \sum_{a \in A_{i}^{+}} f_{a} \mu_{a}=1, i \in I
\end{aligned}
$$

$\mu_{a} \geq 0, a \in A$

$v_{a} \geq 0$

where, $\overline{c_{a t}}$ is the upper bound of passenger flow impedance with terminal point $t$ on edge $a$; $c_{a}$ is the lower bound of passenger flow impedance with terminal point $t$ on edge $a$ $\delta_{a t}=\left\{\begin{array}{cc}1 & \text { if the passenger passes through a } \\ 0 & \text { otherwise }\end{array}\right.$

\subsection{Algorithm}

In this paper, the method of successive averages (MSA) is used to solve the model. MSA algorithm is an iterative algorithm, which uses the weighted average of multiple cycles to get the final solution assigned on each path. Each cycle can get a group of additional bus passenger flow. The weighted average of the flow and the assigned flow is used as the assigned solution of the next cycle. MSA algorithm is simple, easy to program, and has good convergence under the premise of ensuring accuracy. The following Fig. 3 shows the MSA algorithm flow of the model constructed in this paper.

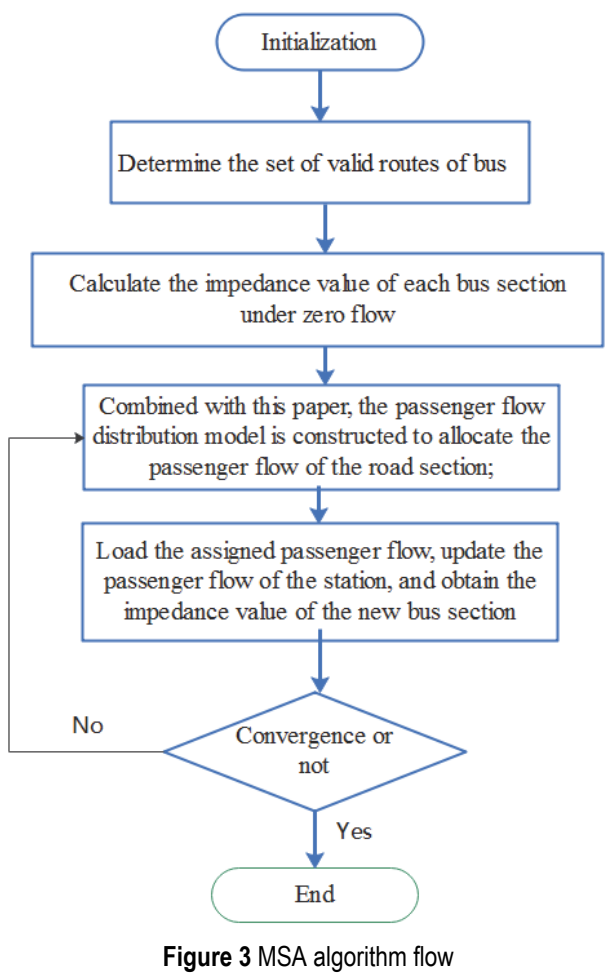

The calculation steps are as follows:

Step 1: determining the effective path set. According to the constraints of feasible path and effective path, the effective path search method constructed in reference [23] is used to obtain the effective path set of public transport travel.

Step 2: initialization. The impedance value of each bus section under zero flow state is calculated.

Step 3: assigning the passenger flow of the road section;

Step 4: load the assigned passenger flow, update the passenger flow of the station, and obtain the impedance value of the new bus section;

Step 5: convergence test.

\section{NUMERICAL EXAMPLES \\ 4.1 Data}

Gongming central area in Guangming District of Shenzhen city is selected as the research area. The area is surrounded by Minsheng Avenue Jimei Road Changchun middle road Songbai road Gongming east ring Avenue, covering an area of about $2.25 \mathrm{~km}^{2}$.

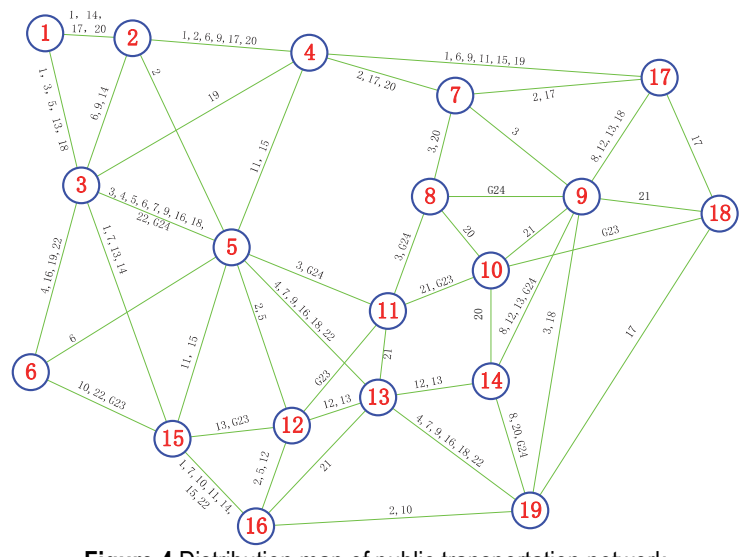

Figure 4 Distribution map of public transportation network 
There are schools, hospitals, commercial, technological industries, residential areas and other types of land in the area, as shown in Fig. 4.

Select the bus IC card data and bus GPS data for seven days (one week) from October 14 to 20, 2019 to obtain the peak hour operation time between stations, after data fusion, we take their quartile value. The bus lines and their numbers at stations are shown in Tab. 1, the departure frequency of bus lines is shown in Tab. 2, and the impedance distribution between some stations is shown in Tab. 3, The distribution of peak hour OD traffic among nodes is shown in Tab. 4.

Table 1 Station and line number

\begin{tabular}{|c|c|c|c|}
\hline Station number & Bus line & Station number & Bus line \\
\hline 1 & $1,3,5,13,14,17,18,20$, & 11 & $3,21, \mathrm{G} 23, \mathrm{G} 24$ \\
\hline 2 & $1,2,6,9,14,17$, & 12 & $2,5,12,13, \mathrm{G} 23$, \\
\hline 3 & $1,3,4,5,6,7,9,13,14,16,18,19,22, \mathrm{G} 24$ & 13 & $4,9,12,13,16,18,21,22$, \\
\hline 4 & $1,2,6,9,11,15,17,19,20$, & 14 & $8,12,13,20, \mathrm{G} 24$ \\
\hline 5 & $2,3,4,5,6,7,9,11,15,16,18,22, \mathrm{G} 24$ & 15 & $1,7,10,11,13,14,15,22, \mathrm{G} 23$, \\
\hline 6 & $4,6,10,16,19,22, \mathrm{G} 23$, & 16 & $1,2,5,7,10,11,12,14,15,21,22$, \\
\hline 7 & $2,3,17,20$ & 17 & $1,2,6,8,9,11,12,13,15,17,18,19$, \\
\hline 8 & $3,20, \mathrm{G} 24$ & 18 & $17,21, \mathrm{G} 23$, \\
\hline 9 & $3,8,12,13,18,21, \mathrm{G} 24$ & 19 & $2,3,4,7,8,9,10,16,17,18,20,22, \mathrm{G} 24$ \\
\hline
\end{tabular}

Table 2 Bus line departure frequency

\begin{tabular}{|c|c|c|c|c|c|c|c|c|c|c|}
\hline Line number & Bus line & \multicolumn{8}{|c|}{ Station number which stop at this station } & Departure frequency \\
\hline 1 & 325 & 16 & 15 & 3 & 1 & 2 & 4 & 17 & & 11 \\
\hline 2 & 882 & 19 & 16 & 12 & 5 & 2 & 4 & 7 & 17 & 14 \\
\hline 3 & B720 & 19 & 9 & 7 & 8 & 11 & 5 & 3 & 1 & 12 \\
\hline 4 & B868 & 6 & 3 & 5 & 13 & 19 & & & & 20 \\
\hline 5 & B899 & 16 & 12 & 5 & 3 & 1 & & & & 15 \\
\hline 6 & E14 & 6 & 5 & 3 & 2 & 4 & 17 & & & 15 \\
\hline 7 & E15 & 19 & 5 & 3 & 15 & 16 & & & & 21 \\
\hline 8 & M193 & 19 & 14 & 9 & 17 & & & & & 15 \\
\hline 9 & M216 & 17 & 4 & 2 & 3 & 5 & 13 & 19 & & 10 \\
\hline 10 & M218 & 6 & 15 & 16 & 19 & & & & & 12 \\
\hline 11 & M238 & 16 & 15 & 5 & 4 & 17 & & & & 16 \\
\hline 12 & M243 & 16 & 12 & 13 & 14 & 9 & 17 & & & 12 \\
\hline 13 & M256 & 1 & 3 & 15 & 12 & 13 & 14 & 9 & 17 & 12 \\
\hline 14 & M284 & 16 & 15 & 3 & 2 & 1 & & & & 10 \\
\hline 15 & M337 & 16 & 15 & 5 & 4 & 17 & & & & 8 \\
\hline 16 & M410 & 19 & 13 & 5 & 3 & 6 & & & & 10 \\
\hline 17 & M436 & 19 & 18 & 17 & 7 & 4 & 2 & 1 & & 15 \\
\hline 18 & M455 & 1 & 3 & 5 & 13 & 19 & 9 & 17 & & 12 \\
\hline 19 & M490 & 17 & 4 & 3 & 6 & & & & & 18 \\
\hline 20 & M519 & 19 & 14 & 10 & 8 & 7 & 4 & 1 & & 10 \\
\hline 21 & M529 & 16 & 13 & 11 & 10 & 9 & 18 & & & 10 \\
\hline 22 & M549 & 19 & 13 & 5 & 3 & 6 & 15 & 16 & & 15 \\
\hline G23 & 35 & 6 & 15 & 12 & 11 & 10 & 18 & & & 10 \\
\hline $\mathrm{G} 24$ & 132 & 19 & 14 & 9 & 8 & 11 & 5 & 3 & & 10 \\
\hline
\end{tabular}

Table 3 Impedance value between some stations, min

\begin{tabular}{|c|c|c|c|c|c|c|c|}
\hline Table 3 Impedance value between some stations, min & Interval value \\
\hline station & station & Bus line & Interval value & station & station & Bus line & {$[2.9,4.6]$} \\
\hline 16 & 15 & 1 & {$[3.7,5.1]$} & 15 & 12 & 13 & {$[2.2,3.1]$} \\
\hline 15 & 3 & 1 & {$[5.4,7.9]$} & 12 & 13 & 13 & {$[2.7,4.2]$} \\
\hline 3 & 1 & 1 & {$[2.4,3.8]$} & 13 & 14 & 13 & {$[4.9,6.1]$} \\
\hline 1 & 2 & 1 & {$[3.8,6.2]$} & 14 & 9 & 13 & {$[2.4,3.6]$} \\
\hline 2 & 4 & 1 & {$[4.1,6.7]$} & 9 & 17 & 13 & {$[3.7,5.1]$} \\
\hline 4 & 17 & 1 & {$[4.3,6.9]$} & 16 & 15 & 14 & {$[5.4,7.9]$} \\
\hline 19 & 16 & 2 & {$[6.5,7.6]$} & 15 & 3 & 14 & {$[2.7,3.9]$} \\
\hline 16 & 12 & 2 & {$[2.4,3.6]$} & 3 & 2 & 14 & {$[3.8,6.2]$} \\
\hline 12 & 5 & 2 & {$[4.2,5.6]$} & 2 & 1 & 14 & {$[3.7,5.1]$} \\
\hline 5 & 2 & 2 & {$[3.8,5.3]$} & 16 & 15 & 15 & {[} \\
\hline
\end{tabular}

Table 4 Peak hour OD flow, person/hour

\begin{tabular}{|c|c|c|c|c|c|c|c|c|c|c|c|c|c|c|c|c|c|c|c|}
\hline node & 1 & 2 & 3 & 4 & 5 & 6 & 7 & 8 & 9 & 10 & 11 & 12 & 13 & 14 & 15 & 16 & 17 & 18 & 19 \\
\hline 1 & 0 & 2 & 4 & 18 & 9 & 14 & 12 & 9 & 10 & 11 & 13 & 12 & 6 & 8 & 8 & 29 & 25 & 6 & 14 \\
\hline 2 & 2 & 0 & 6 & 7 & 5 & 14 & 9 & 5 & 6 & 4 & 7 & 14 & 5 & 4 & 10 & 12 & 17 & 7 & 9 \\
\hline 3 & 4 & 6 & 0 & 7 & 4 & 6 & 10 & 9 & 8 & 9 & 19 & 14 & 25 & 9 & 10 & 28 & 19 & 13 & 24 \\
\hline 4 & 18 & 7 & 7 & 0 & 6 & 11 & 4 & 2 & 3 & 11 & 12 & 14 & 12 & 9 & 13 & 10 & 5 & 12 & 11 \\
\hline 5 & 9 & 5 & 4 & 6 & 0 & 5 & 5 & 4 & 9 & 11 & 7 & 7 & 9 & 17 & 7 & 22 & 20 & 15 & 30 \\
\hline 6 & 14 & 14 & 6 & 11 & 5 & 0 & 8 & 7 & 6 & 7 & 9 & 12 & 13 & 10 & 3 & 7 & 8 & 11 & 13 \\
\hline 7 & 12 & 9 & 10 & 4 & 5 & 8 & 0 & 3 & 3 & 6 & 8 & 9 & 7 & 11 & 6 & 13 & 3 & 8 & 8 \\
\hline 8 & 9 & 5 & 9 & 2 & 4 & 7 & 3 & 0 & 4 & 2 & 3 & 7 & 8 & 12 & 14 & 17 & 7 & 11 & 12 \\
\hline 9 & 10 & 6 & 8 & 3 & 9 & 6 & 3 & 4 & 0 & 5 & 8 & 15 & 16 & 5 & 15 & 17 & 6 & 6 & 7 \\
\hline 10 & 11 & 4 & 9 & 11 & 11 & 7 & 6 & 2 & 5 & 0 & 6 & 10 & 11 & 5 & 18 & 15 & 10 & 5 & 17 \\
\hline 11 & 13 & 7 & 19 & 12 & 7 & 9 & 8 & 3 & 8 & 6 & 0 & 7 & 3 & 6 & 6 & 18 & 22 & 14 & 16 \\
\hline
\end{tabular}


Table 4 Peak hour OD flow, person/hour - continuous

\begin{tabular}{|c|c|c|c|c|c|c|c|c|c|c|c|c|c|c|c|c|c|c|c|}
\hline node & 1 & 2 & 3 & 4 & 5 & 6 & 7 & 8 & 9 & 10 & 11 & 12 & 13 & 14 & 15 & 16 & 17 & 18 & 19 \\
\hline 12 & 12 & 14 & 14 & 14 & 7 & 12 & 9 & 7 & 15 & 10 & 7 & 0 & 5 & 19 & 4 & 5 & 28 & 15 & 17 \\
\hline 13 & 6 & 5 & 25 & 12 & 9 & 13 & 7 & 8 & 16 & 11 & 3 & 5 & 0 & 7 & 8 & 7 & 15 & 7 & 6 \\
\hline 14 & 8 & 4 & 9 & 9 & 17 & 10 & 11 & 12 & 5 & 5 & 6 & 19 & 7 & 0 & 13 & 8 & 14 & 9 & 4 \\
\hline 15 & 8 & 10 & 10 & 13 & 7 & 3 & 6 & 14 & 15 & 18 & 6 & 4 & 8 & 13 & 0 & 3 & 11 & 8 & 10 \\
\hline 16 & 29 & 12 & 28 & 10 & 22 & 7 & 13 & 17 & 17 & 15 & 18 & 5 & 7 & 8 & 3 & 0 & 15 & 7 & 5 \\
\hline 17 & 25 & 17 & 19 & 5 & 20 & 8 & 3 & 7 & 6 & 10 & 22 & 28 & 15 & 14 & 11 & 15 & 0 & 4 & 13 \\
\hline 18 & 6 & 7 & 13 & 12 & 15 & 11 & 8 & 11 & 6 & 5 & 14 & 15 & 7 & 9 & 8 & 7 & 4 & 0 & 6 \\
\hline 19 & 14 & 9 & 24 & 11 & 30 & 13 & 8 & 12 & 7 & 17 & 16 & 17 & 6 & 4 & 10 & 5 & 13 & 6 & 0 \\
\hline
\end{tabular}

\subsection{Bus Passenger Flow Assignment Results}

The results of bus passenger flow assignment under OD and impedance are invariant values and are shown in Fig. 5. In the figure, the flow between each bus node section is the total flow from the section to each terminal.

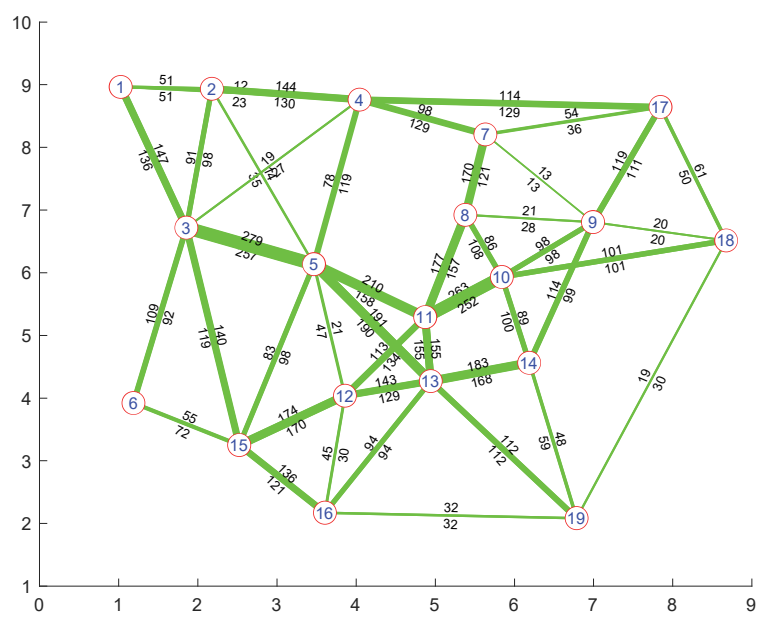

Figure 5 The results of bus passenger flow assignment under $\mathrm{OD}$ and impedance are invariant values

According to the bus passenger flow assignment method in this paper, the bus passenger flow assignment results with OD as the determined value and impedance as the interval value are shown in Fig. 6.

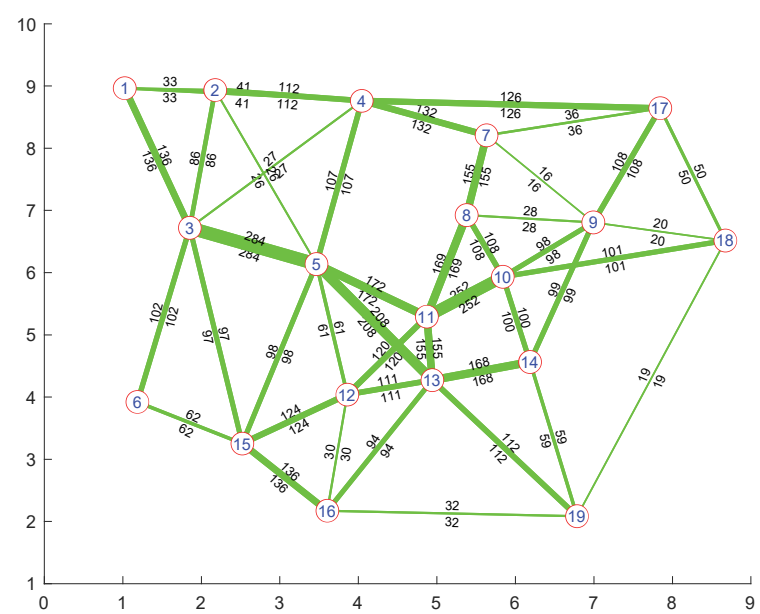

Figure 6 The results of bus passenger flow assignment under OD is determined value and impedance are invariant value

\subsection{Error Analysis}

The mean absolute percentage error (MAPE) and root mean square error (RMSE) are three typical indexes used for evaluating the accuracy of prediction (Eqs. (1) to (3)).

$$
\begin{aligned}
& \text { MAPE }=\frac{1}{n} \sum_{i=1}^{n}\left|\frac{y_{i}-\hat{y}_{i}}{y_{i}}\right| \times 100 \% \\
& R M S E=\sqrt{\frac{1}{n} \sum_{i=1}^{n}\left(\hat{y}_{i}-y_{i}\right)^{2}}
\end{aligned}
$$

where, $y=\left\{y_{1}, y_{2}, \ldots, y_{i}, \ldots y_{n}\right\}$ the sequence of the observation values is, $\hat{y}=\left\{\hat{y}_{1}, \hat{y}_{2}, \ldots, \hat{y}_{i}, \ldots \hat{y}_{n}\right\}$ is the sequence of the prediction values, and $n$ is the number of observation values. In this study, the sample size $n$ equals was 44 .

Comparing the above passenger flow assignment results with the actual bus passenger flow, the error is shown in Table 5. The actual bus passenger flow value can be obtained by the method of deriving the bus passenger travel path after the fusion of bus IC card data and GPS data [24]. The actual value is from October 14 to 20, 2019, including two non-working days (10.19 and 10.20).

Table 5 Comparison of the error between the determined value impedance and the interval uncertainty impedance in the result of bus passenger flow assignment

\begin{tabular}{|c|c|c|c|c|}
\hline \multirow{2}{*}{ Date } & \multicolumn{2}{|c|}{$\begin{array}{c}\text { the determined value } \\
\text { impedance }\end{array}$} & \multicolumn{2}{c|}{$\begin{array}{c}\text { the interval uncertainty } \\
\text { impedance }\end{array}$} \\
\cline { 2 - 5 } & $R M S E$ & $M A P E / \%$ & $R M S E$ & $M A P E / \%$ \\
\hline Oct 14 & 10.2 & 8.3 & 6.8 & 5.2 \\
\hline Oct 15 & 8.3 & 11.5 & 10.2 & 9.3 \\
\hline Oct 16 & 9.5 & 12.1 & 9.7 & 12.8 \\
\hline Oct 17 & 8.6 & 8.4 & 13.3 & 9.6 \\
\hline Oct 18 & 11.5 & 7.2 & 6.9 & 5.5 \\
\hline Oct 19 & 21.2 & 15.6 & 7.5 & 8.8 \\
\hline Oct 20 & 16.7 & 13.5 & 12.6 & 9.8 \\
\hline Average & 12.3 & 10.9 & 9.5 & 8.7 \\
\hline
\end{tabular}

As shown in Tab. 5, the average absolute percentage error is $8.7 \%$ compared with the actual results by using the bus passenger flow distribution model under the interval impedance, and the average absolute percentage error is $10.9 \%$ when the impedance is determined, The result of passenger flow assignment under interval impedance is obviously better than that under invariant impedance. For non-working days, when the bus passenger flow changes greatly, the bus passenger flow assignment result under interval impedance is better. Because taking into account more impedance influence factors with the interval impedance value, although the invariant assignment result may not be as good as the assignment result under certain impedance, the overall accuracy of passenger flow assignment is higher.

\section{CONCLUSIONS}

(1) Combined with the interval uncertainty theory and the basic algorithm of interval number, the OD matrix of 
bus stop boarding and alighting is an interval value, which is more in line with the actual situation.

(2) This paper builds the bus passenger flow assignment model of interval impedance, and designs MSA algorithm to solve it.

(3) The bus impedance interval value of the bus line is obtained through the data mining and processing of Shenzhen bus IC card data and GPS data. The bus impedance of different time periods can be obtained, and then the bus passenger flow of different time periods can be assigned according to the OD matrix.

(4) The error analysis compares the average error and the maximum error, which can better reflect that the assignment model under interval impedance has smaller error and is more suitable for the bus passenger flow assignment under the condition of uncertain boarding and alighting interval.

A future study may develop a transit assignment approach by modeling the feature of morning commute [25, 26] and taking connected and autonomous vehicle environment into account [27-29].

\section{Acknowledgements}

The project was supported by Open Fund of Engineering Research Center of Catastrophic Prophylaxis and Treatment of Road \& Traffic Safety of Ministry of Education (Grant Nos. kfj180401, Changsha University of Science \& Technology); Natural Science Foundation of Hunan Province, China (Grant Nos. 2019JJ40306); The National Natural Science Foundation of China (Grant Nos. 61773077).

\section{REFERENCES}

[1] Dial, R. B. (1967). Transit pathfinder algorithm. Highway Research Record, 205, 67-85.

[2] Dial, R. B. (1971). A probabilistic multipath traffic assignment model which obviates path enumeration. Transportation research, 5(2), 83-111.

[3] Chriqui, C. \& Robillard, P. (1975). Common bus lines. Transportation science, 9(2), 115-121. https://doi.org/10.1287/trsc.9.2.115

[4] De Cea, J. \& Fernández, E. (1993). Transit assignment for congested public transport systems: an equilibrium model. Transportation science, 27(2), 133-147. https://doi.org/10.1287/trsc.27.2.133

[5] Lam, W. H., Zhou, J., \& Sheng, Z. H. (2002). A capacity restraint transit assignment with elastic line frequency. Transportation Research Part B: Methodological, 36(10), 919-938. https://doi.org/10.1016/S0191-2615(01)00042-X

[6] Cominetti, R. \& Correa, J. (2001). Common-lines and passenger assignment in congested transit networks. Transportation science, 35(3), 250-267. https://doi.org/10.1287/trsc.35.3.250.10154

[7] Cepeda, M., Cominetti, R., \& Florian, M. (2006). A frequency-based assignment model for congested transit networks with strict capacity constraints: characterization and computation of equilibria. Transportation research part $B$ : Methodological, 40(6), 437-459. https://doi.org/10.1016/j.trb.2005.05.006

[8] Poon, M. H., Wong, S. C., \& Tong, C. O. (2004). A dynamic schedule-based model for congested transit networks. Transportation Research Part B: Methodological, 38(4), 343368. https://doi.org/10.1016/S0191-2615(03)00026-2
[9] Hamdouch, Y., Ho, H. W., Sumalee, A., \& Wang, G. (2011). Schedule-based transit assignment model with vehicle capacity and seat availability. Transportation Research Part B: Methodological, 45(10), 1805-1830. https://doi.org/10.1016/j.trb.2011.07.010

[10] Hamdouch, Y. \& Lawphongpanich, S. (2008). Schedulebased transit assignment model with travel strategies and capacity constraints. Transportation Research Part B: Methodological, 42(7-8), 663-684. https://doi.org/10.1016/j.trb.2007.11.005

[11] Zhang, Y., Lam, W. H., Sumalee, A., Lo, H. K., \& Tong, C. O. (2010). The multi-class schedule-based transit assignment model under network uncertainties. Public Transport, 2(1-2), 69-86. https://doi.org/10.1007/s12469-010-0027-4

[12] Codina, E. (2013). A variational inequality reformulation of a congested transit assignment model by Cominetti, Correa, Cepeda, and Florian. Transportation Science, 47(2), 231-246. https://doi.org/10.1287/trsc.1120.0427

[13] Cominetti, R. \& Correa, J. (2001). Common-lines and passenger assignment in congested transit networks. Transportation science, 35(3), 250-267. https://doi.org/10.1287/trsc.35.3.250.10154

[14] Bouzaïene-Ayari, B., Gendreau, M., \& Nguyen, S. (2001). Modeling bus stops in transit networks: A survey and new formulations. Transportation Science, 35(3), 304-321. https://doi.org/10.1287/trsc.35.3.304.10148

[15] Sun, S. \& Szeto, W. Y. (2018). Logit-based transit assignment: Approach-based formulation and paradox revisit. Transportation Research Part B: Methodological, 112, 191215. https://doi.org/10.1016/j.trb.2018.03.018

[16] Gilbert, H. \& Spanjaard, O. (2017). A double oracle approach to minmax regret optimization problems with interval data. European Journal of Operational Research, 262(3), 929-943. https://doi.org/10.1016/j.ejor.2017.04.058

[17] Batarseh, O. G. \& Wang, Y. (2018). An interval-based approach to model input uncertainty in $\mathrm{M} / \mathrm{M} / 1$ simulation. International Journal of Approximate Reasoning, 95, 46-61. https://doi.org/10.1016/j.ijar.2017.12.007

[18] Zaidi, S. \& Kroll, A. (2017). NOE TS fuzzy modelling of nonlinear dynamic systems with uncertainties using symbolic interval-valued data. Applied Soft Computing, 57, 353-362. https://doi.org/10.1016/j.asoc.2017.04.004

[19] Liu, W., Tan, Q., \& Wu, W. (2020). Forecast and Early Warning of Regional Bus Passenger Flow Based on Machine Learning. Mathematical Problems in Engineering, 2020. https://doi.org/10.1155/2020/6625435

[20] Soto, A. \& Friesz, T. L. (2003). Estrous: A computer package for solving supply-demand equilibrium problems on multimodal urban transportation networks with multiple user classes. Proceedings of the 82 th transportation research board(TRB) annual meeting, 1-33.

[21] Charnes, A. \& Cooper, W. W. (1959). Chance-constrained programming. Management science, 6(1), 73-79. https://doi.org/10.1287/mnsc.6.1.73

[22] Yaman, H., Karaşan, O. E., \& Pınar, M. C. (2001). The robust spanning tree problem with interval data. Operations research letters, 29(1), 31-40. https://doi.org/10.1016/S0167-6377(01)00078-5

[23] Feng, X., Zhou, H. P., \& Peng, W. (2018). Robust shortest path algorithm based on Benders decomposition. Journal of Changsha University of Science and Technology(Natural Science), 15(02), 16-22.

[24] Liu, W., Tan, Q., \& Liu, L. (2020). Destination Estimation for Bus Passengers Based on Data Fusion. Mathematical Problems in Engineering, 2020. https://doi.org/10.1155/2020/8305475

[25] Wu, W., Liu, W., Zhang, F., \& Dixit, V. (2021). A New Flexible Parking Reservation Scheme for the Morning Commute under Limited Parking Supplies. Networks and Spatial Economics, 1-33. 
https://doi.org/10.1007/s11067-021-09538-5

[26] Xiong, X. X., Zhang, S. C., \& Guo, L. (2021). Non-motorized vehicle traffic accidents in China: Analysing Road users' precrash behaviors and implications for road safety. International Journal of Safety and Security Engineering, 11(1), 105-116. https://doi.org/10.18280/ijsse.110112

[27] Wu, W., Zhang, F., Liu, W., \& Lodewijks, G. (2020). Modelling the traffic in a mixed network with autonomousdriving expressways and non-autonomous local streets. Transportation Research Part E: Logistics and Transportation Review, 134, 101855. https://doi.org/10.1016/j.tre.2020.101855

[28] Karyemsetty, N. \& Kumar, K. R. (2020). Road safety: An accident prevention using intelligent vehicular network. International Journal of Safety and Security Engineering, 10(5), 631-638. https://doi.org/10.18280/ijsse.100507

[29] Youssef, F. \& Houda, B. (2019). Optimal combination of imitation and reinforcement learning for self-driving cars. Revue d'Intelligence Artificielle, 33(4), 265-273.

https://doi.org/10.18280/ria.330402

\section{Contact information:}

\section{Qian TAN}

School of Traffic \& Transportation Engineering, Central South University,

Changsha 410075, China

E-mail: tq.helen@csu.edu.cn

\section{Xiangdong ZHOU}

(Corresponding author)

Beijing qianfang Technology Co., Ltd,

Beijing 100085, China

E-mail: 20101030050@stu.csust.edu.cn

\section{Wusheng LIU}

School of Traffic and Transportation Engineering,

Changsha University of Science \& Technology,

Changsha 410114, China

E-mail: Iwusheng@csust.edu.cn 